\title{
Die Wirkung des Wassers - Hydrotherapie beim Kleintier
}

\author{
Stefanie Süße, Reinhold Scharwey
}

Wasser kann mit seinem positiven Einfluss auf den Organismus für unterschiedliche therapeutische Indikationen wirkungsvoll genutzt werden. Doch welche Behandlungsformen finden beim Kleintier Anwendung und worauf sollte man bei Hund und Katze achten?

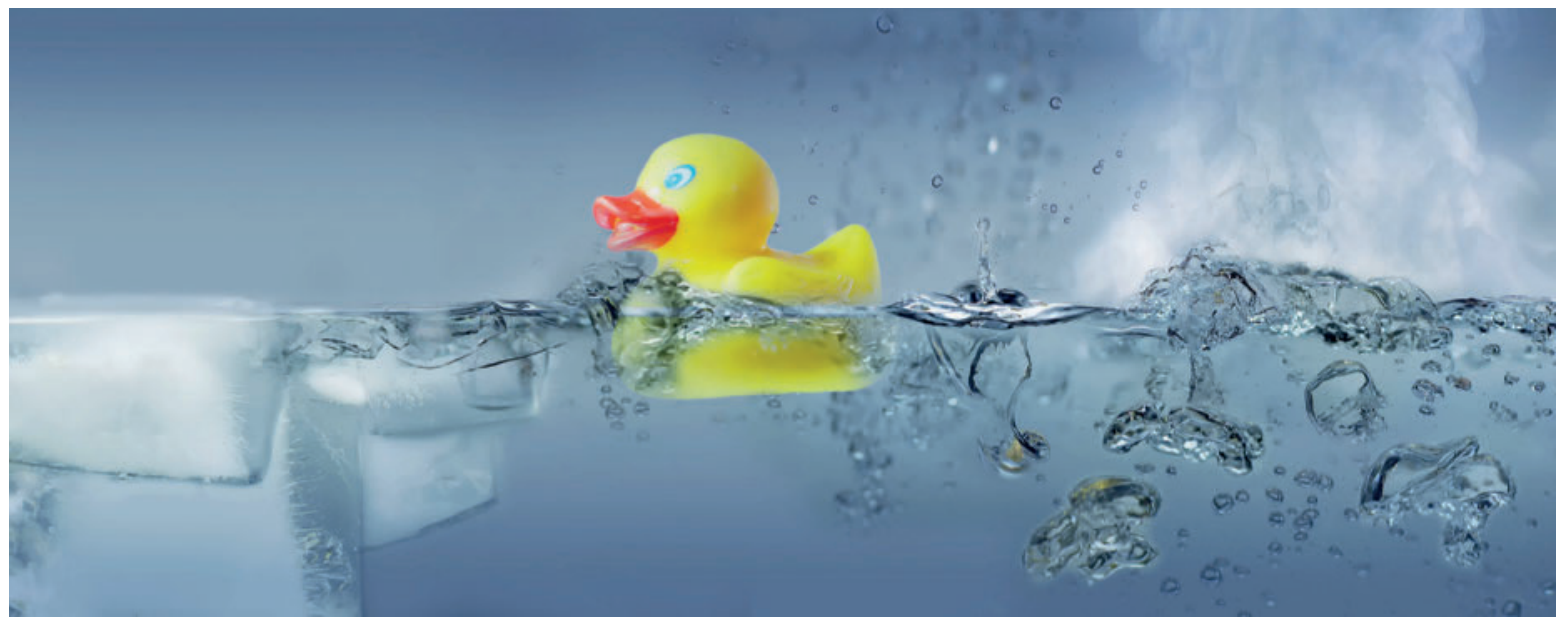

Heiß oder kalt. Fest, flüssig oder gasförmig. So vielfältig wie die Formen des Wassers sind auch dessen Anwendungsgebiete und -möglichkeiten in der Tierphysiotherapie. $\odot$ mpix-foto-adobe.stock.com [rerif]

Hydrotherapie ist die methodische Anwendung von Wasser in seinen verschiedenen Aggregatzuständen zu prophylaktischen, therapeutischen und diätetischen Zwecken (Wilhelm Winternitz, $\left.{ }^{*} 1.3 .1834\right)$.

\section{Grundlagen, die man wissen sollte}

\section{Hydrostatischer Druck}

Flüssigkeiten üben auf eingetauchte Körper Druck aus. Der sogenannte hydrostatische Druck wirkt also auch komprimierend auf die Venen. Hierdurch wird die Blutmenge im Kreislauf erhöht, es kommt zum Blutdruckanstieg. Der Anstieg des Blutdrucks verhält sich dabei proportional zur Eintauchtiefe. Ist der gesamte Körper unter Wasser, so wirkt der hydrostatische Druck auch auf den Bauch- und Brustraum komprimierend. Nach Strasburger wird der Brustkorb bei Menschen in einem Wannenbad um 1,0-3,5 cm und der Bauchumfang um $2,5-6,5 \mathrm{~cm}$ verringert. Die Einatmung wird durch die komprimierende Wirkung deutlich erschwert und kann, wenn gewünscht, in dieser Form trainiert werden (Atemtraining).

\section{CAVE}

Herz-Kreislauf-Erkrankungen und Atemwegbeschwerden sind Kontraindikation für die Hydrotherapie. Bei leichteren Herzerkrankungen und gut eingestellten Herzpatienten ist die Therapie bei Bedarf möglich, die Patienten müssen bei der Anwendung aber gut beobachtet werden. Deutet das Verhalten des Tieres auf Unwohlsein hin, ist die Therapie sofort abzubrechen.

\section{Auftriebskraft}

Das archimedische Prinzip besagt, dass ein in eine Flüssigkeit eingetauchter Körper scheinbar so viel an Gewicht verliert, wie das von ihm verdrängte Flüssigkeitsmaß wiegt. Der lebende Körper wird fast Schwerelos. Es handelt sich 
dabei um einen passiven Vorgang. Messungen nach David Levine ergaben unterschiedliche Reduzierungen des Gewichts, je nach Wasserstand. Die Auftriebskraft von Flüssigkeiten kann daher in der Physiotherapie gezielt zur Entlastung der Gelenke genutzt werden. Dies spielt vor allen bei Erkrankungen der Gelenke bei Hunden (und Katzen) mit und ohne Übergewicht eine Rolle. Bei einem Wasserstand bis zum Sprunggelenk reduziert sich das Gewicht um ca. 9\%. Wird der Körper bis zum Knie eingetaucht, reduziert sich das Gewicht schon um 15\% und bei einem Wasserstand bis zur Hüfte um ca. 62\%.

\section{KATZEN IN DER HYDROTHERAPIE}

Entgegen unserer ursprünglichen Meinung, Katzen seien nicht für die Hydrotherapie geeignet, konnten wir zeigen, dass Katzen sogar das Unterwasserlaufband tolerieren.

\section{Reibungswiderstand}

Bewegung im Wasser wird durch den Reibungswiderstand erschwert. Je schneller die Bewegung stattfindet, umso stärker ist der Reibungswiderstand. Der Reibungswiderstand wird therapeutisch zum Muskelaufbau genutzt, z. B. beim Laufen im Wasser.

\section{Metazentrisches Prinzip}

In Flüssigkeiten schwimmende Körper werden durch 2 entgegengesetzte Kräfte beeinflusst. Die Gravitation richtet ihre Kraft gegen den Auftrieb der Flüssigkeit. Der Schwimmpunkt eines Körpers (Metazentrum) ist der Punkt, an dem ein ausgeglichenes Verhältnis zwischen Gravitation bzw. Masse des Körpers und Auftrieb herrscht. Gleichgewichtsreaktionen im Wasser können sehr gut zur Koordinationsschulung genutzt werden.

\section{Temperatur}

Ein lebender Körper empfindet Temperaturunterschiede im Verhältnis zu seiner eigenen Hauttemperatur. Die Temperatur wird in 4 Bereiche unterteilt ( $\vee$ Tab.1). Die Wärmeleitfähigkeit des umgebenden Mediums ist ebenso von Bedeutung. Wasser hat eine sehr gute Wärmeleitfähigkeit. Höhere Temperaturen werden als warm oder heiß empfunden, geringere Temperaturen werden als kalt empfunden.
Die unterschiedliche Wärmeleitfähigkeit der Luft und des Wassers bedingen die entsprechenden Unterschiede in der Wahrnehmung. Bei der sogenannten indifferenten Temperatur wird keine thermische Veränderung wahrgenommen. An der Luft beträgt die indifferente Temperatur in der Regel $22-24^{\circ} \mathrm{C}$, im Wasser $34-36^{\circ} \mathrm{C}$. Entscheidend ist aber die tatsächliche Hauttemperatur. Das bedeutet, die indifferente Temperatur ist individuell. Als kalt werden Temperaturen unter der indifferenten Temperatur wahrgenommen, als warm bis $2^{\circ} \mathrm{C}$ darüber. Gefühlt heiß wahrgenommen werden Temperaturen über der Warmempfindung.

Die indifferente Temperatur ist auf den Körper wirkungslos. Kalte Temperaturen bewirken eine Gefäßkonstriktion mit einer darauffolgenden Gefäßdilatation. Die Reaktionsstärke ist von der Temperaturdifferenz abhängig. Je höher die Differenz, umso heftiger folgt die Reaktion. Warme Temperaturen bewirken eine sofortige Gefäßdilatation. Heiße Temperaturen bewirken eine Gefäßkonstriktion mit einer darauffolgenden Gefäßdilatation und einer Kapillarisation.

Unter einer konsensuellen Reaktion sieht man das gleichsinnige Mitreagieren der kontralateralen Körperseite in abgeschwächter Form.

\section{CAVE}

Paradoxe Gefäßreaktionen, d. h. auftretende Gefäßspasmen nach Heiß- oder Kaltanwendungen, sind bei humanen Patienten mit vorgeschädigten Gefäßen beobachtet worden. Aufgrund dieser Erfahrungen sollte man auch beim Tier auf

Schmerzreaktionen während der Anwendung achten, gegebenenfalls die Behandlung abbrechen und für eine Thermoregulation sorgen.

\section{Was sonst noch reizt}

\section{Chemische Reize}

Chemische Reize können durch Zusatzstoffe (z. B. Salze, Öle, Duftstoffe) ausgelöst werden, die dem Wasser zugegeben werden. Sie können hilfreich sein, sind aber auch bei unerwünschten Reaktionen (z. B. Hautreaktionen) zu berücksichtigen.

- Tab.1 Einteilung der Temperaturwahrnehmung an der Luft und im Wasser, zu berücksichtigen ist die eigene Hauttemperatur.

\begin{tabular}{|c|c|c|c|}
\hline Temperaturwahrnehmung & Luft ${ }^{\circ} \mathrm{C}$ & Wasser ${ }^{\circ} \mathrm{C}$ & \\
\hline indifferente Temperatur & $22-24$ & $34-36$ & \multirow{4}{*}{$\begin{array}{l} \pm \text { durch individuelle } \\
\text { Hauttemperatur! }\end{array}$} \\
\hline kalt & $<22-24$ & $<34-36$ & \\
\hline warm & $>22-24$ und $<24-26$ & $>34-36$ und $<36-38$ & \\
\hline heiß & $>24-26$ & $>36-38$ & \\
\hline
\end{tabular}




\section{Mechanische Reize}

In der Druckstrahlanwendung oder bei der Nutzung von Gegenstromanlagen können zusätzlich mechanische Reize zum Einsatz kommen. Im Bereich der Elektrotherapie (Ultraschall) kann Wasser als Kopplungsmedium zur Behandlung von Zehengelenken gut eingesetzt werden (subaquale Anwendung des therapeutischen Ultraschalls).

\section{Elektrische Reize}

Im Bereich der Elektrotherapie kann Wasser als Kopplungsmedium zur Behandlung von Zehengelenken gut eingesetzt werden (subaquale Anwendung von Einzelelektroden im Rahmen der Interferenzstrom-Regulationstherapie.).

\section{Behandlungsformen}

\section{Kneipp`sche Güsse}

Bei einer Gussanwendung nach Kneipp soll der Guss drucklos und als gebundene Wasserstrahlanwendung durchgeführt werden, optimal mit brunnenkaltem Wasser. Da brunnenkaltes Wasser selten zur Verfügung steht, genügt kaltes Wasser aus der Leitung. Dabei soll das Wasser als Mantel über den Körper ablaufen. Während der Anwendung soll der Patient nicht im Wasser stehen, es muss also für einen Wasserablauf gesorgt sein. Grundsätzlich wird der Kneipp'sche Guss von peripher nach zentral und wieder zurück nach peripher durchgeführt. Der Guss nach zentral sollte dabei langsam erfolgen, wobei der Guss nach peripher in der Geschwindigkeit des ablaufenden Wassers stattfindet. Die Gussfolge wird 3-mal in dieser Art wiederholt. Bei stark befellten Tieren sollte das Fell vor der Anwendung mit indifferent temperiertem Wasser durchnässt werden.
Indikationen für die verschiedenen Kneipp'schen Güsse sind:

- vegetative Umstimmung

- Blutdruckregulation

- Durchblutungsförderung durch Vasodilatation

- funktionelle Organstörungen im Sinne einer Regulation

\section{Guss der Hinterextremitäten}

Dieser Guss beginnt aufgrund der geringeren Herz-Kreislauf-Belastung herzfern an der Außenseite der rechten Pfote ( $\triangleright$ Abb. 1). Der von peripher nach zentral gerichtete drucklose Strahl sollte bis zum Trochanter major aufsteigen, hier kurz innehalten und anschließend über die Glutealmuskulatur nach kaudal gerichtet werden. Der sich anschließende nach peripher gerichtete Guss wird über die kaudalen Adduktoren bis zur Pfote geführt. Die linke Hinterextremität wird anschließend in gleicher Weise behandelt. Dieser Ablauf wird insgesamt 3-mal durchgeführt. Die gesamte Anwendung sollte maximal 2 Minuten dauern.

\section{Guss der Vorderextremitäten}

Der Guss wird aufgrund der geringeren Herz-KreislaufBelastung herzfern an der rechten Vorderpfote peripher begonnen und über die reizunempfindlichere Außenseite und den M. triceps brachii nach proximal bis auf Höhe des oberen Randes des Schulterblatts geführt und hier kurz innegehalten. Der absteigende Guss erfolgt über den M. biceps brachii nach medial und distal zurück zur Pfote. Die linke Vorderextremität wird in gleicher Weise behandelt. Dieser Ablauf wird insgesamt 3-mal durchgeführt. Die gesamte Anwendung sollte maximal 2 Minuten dauern.
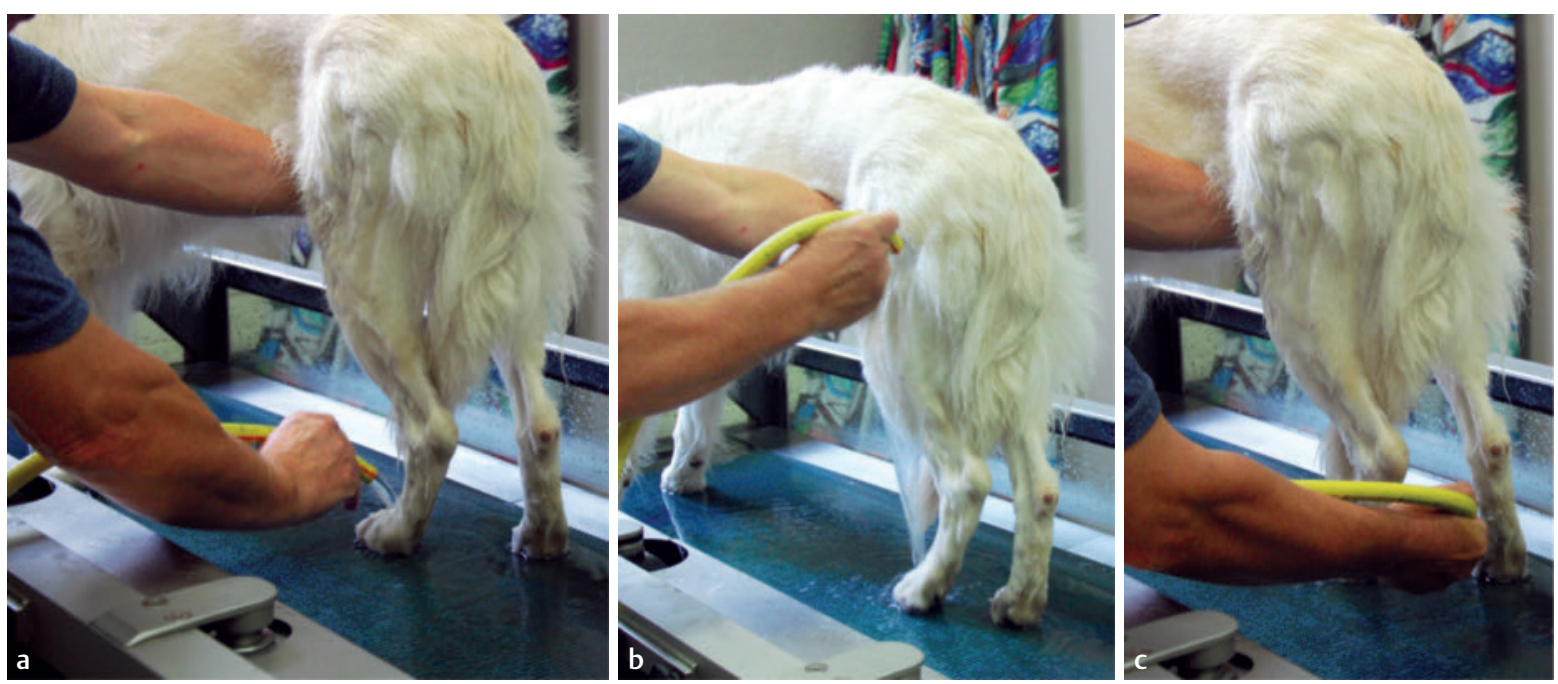

- Abb.1 Guss der linken Hintergliedmaße bei einer 10-jährigen weißen Schäferhündin. Der Guss wird außen an der Hinterpfote beginnend bis zum Trochanter major und dann über die Glutealmuskulatur nach kaudal geführt. Von dort wird er über die kaudalen Adduktoren nach peripher bis zur Pfote geführt. Quelle: @ Stefanie Süße, Reinhold Scharwey 


\section{Thoraxguss}

Der Thoraxguss ( $\bullet$ Abb.2) erfolgt aufsteigend an der Außenseite der rechten Vorderpfote über den M. triceps brachii nach proximal bis auf Höhe des oberen Randes des Schulterblatts und anschließend waagerecht nach kaudal auf Höhe des oberen Drittels des Brustkorbs bis maximal zum 13. Brustwirbel. Über den ventralen Anteil des Thorax erfolgt der Rückweg des Gusses waagerecht zurück zur Extremität und dort auf der Innenseite zur Pfote. Die Gegenseite erfolgt gleichermaßen. Die Durchführung des Thoraxgusses erfolgt 3-mal.

\section{Abdomenguss}

Der Guss ( $\vee$ Abb.3) beginnt an der Außenseite der rechten hinteren Pfote. Der von peripher nach zentral gerichtete drucklose Strahl sollte bis zum Trochanter major aufsteigen und anschließend waagerecht über das obere Drittel des Bauchraums bis kurz vor den Brustkorbrand geführt werden. Der Rückweg erfolgt an der Ventralseite des Abdomens zur Hinterextremität und an deren Medialseite zurück zur Pfote. Die Gegenseite erfolgt gleichermaßen. Die Durchführung des Abdomengusses erfolgt 3-mal.

\section{Ganzkörperguss}

Die intensivste vegetative Umstimmung (Regulation) ist mit einem Ganzkörperguss zu erreichen. Die Durchführung entspricht einer Kombination bzw. Zusammensetzung aus der Abdomen- und Thoraxanwendung, dabei beginnt die Anwendung an der Pfote der rechten Hintergliedmaße.

\section{Wickel, Auflagen und Kompressen}

Wickel/Umschläge, Auflagen und Kompressen nach Prießnitz und Kneipp unterscheiden sich grundsätzlich in der Größe der Anwendung. Sie erstrecken sich auf Teilgebiete des Körpers. Wickel (Umschläge) werden

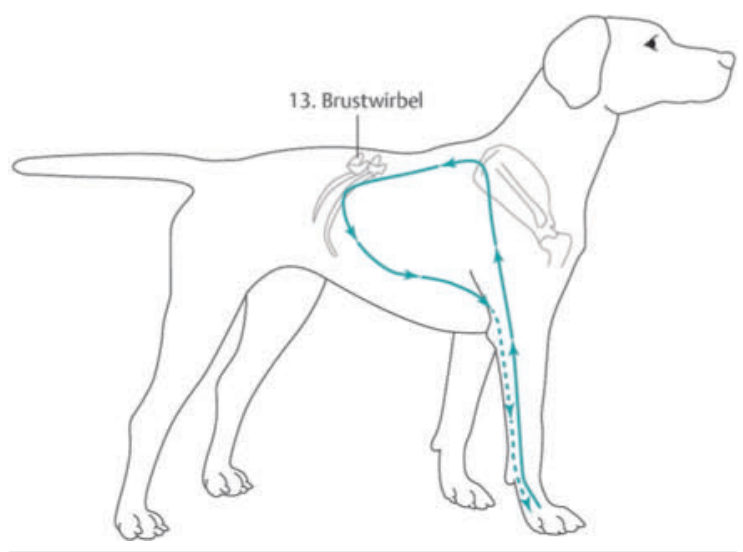

- Abb. 2 Der Thoraxguss beginnt an der Außenseite der rechten Vorderpfote bis zum Schulterblattknorpel, dann waagerecht bis auf Höhe des 13. Brustwirbels. Dann verläuft er über den ventralen Anteil des Thorax zurück zur Extremität und über die Innenseite (gestrichelte Linie) zur Pfote. Quelle: @ Thieme Gruppe

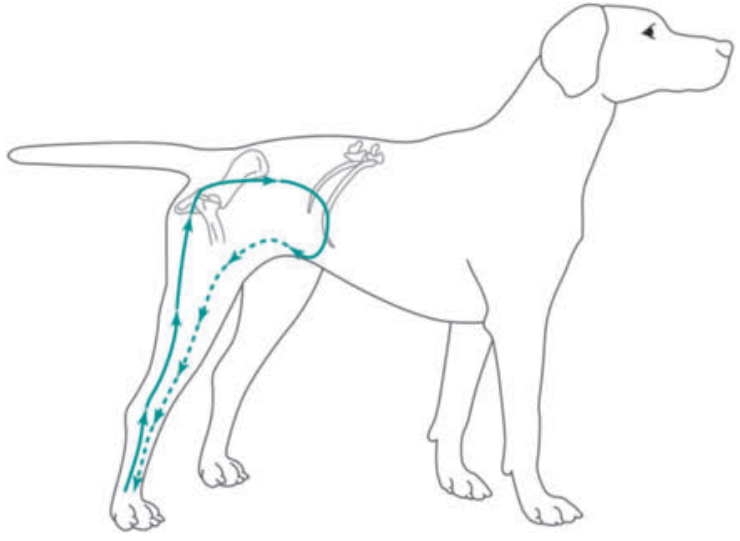

- Abb. 3 Der Abdomenguss beginnt an der Außenseite der rechten Hinterpfote bis zum Trochanter major und verläuft dann waagerecht über das obere Drittel des Bauchraums bis vor den Brustkorb. Nun verläuft er ventral des Abdomens zur Hinterextremität und dort medial (gestrichelte Linie) zur Pfote. Quelle: @ Thieme Gruppe

zirkulär um die Körperteile gelegt. Auflagen und Kompressen werden hingegen nur lokal aufgebracht. Die Kompresse ist die flächenmäßig kleinere Anwendung der Auflage. Je nach Indikation können wärmeentziehende und wärmestauende Wirkungen (z.B. beim PrießnitzUmschlag) zur Anwendung kommen.

Packungen, wie sie in der Humanmedizin genutzt werden, finden im Bereich der Kleintierpraxis keine Anwendung. Sie umfassen beim Menschen den ganzen Körper.

\section{Wärmeentziehender Wickel}

Mögliche Anwendungsbereiche für wärmeentziehende Wickel sind akute hyperthermische Gelenkentzündungen. Einen wärmeentziehenden (kalten) Wickel legt man nur kurz an, mit regelmäßigen Wechseln nach nur wenigen Minuten. Das Nasstuch darf sich nicht erwärmen! Diese Wirkung kann auch durch den Einsatz von Quark, Heilerde ( $\boldsymbol{A} \mathbf{A b}$.4) usw. herbeigeführt werden. Das dazu benötigte Nasstuch wird mit kaltem Wasser getränkt, wichtig ist hierbei die Berücksichtigung der Ausgangstemperatur des Patienten (siehe „Grundlagen, die man Wissen sollte“). Bis zu $10^{\circ} \mathrm{C}$ Differenz unter der Körpertemperatur werden in der Regel nicht als unangenehm empfunden. Nach dem Wickeln oder Aufbringen des Nasstuchs wird dieses anschließend mit einem Trockentuch umwickelt bzw. belegt. Um die isolierende Wirkung des Felles bestmöglich zu reduzieren, sollte stärker ausgeprägtes Haarkleid, vor Anlegen des Nasstuchs, an den entsprechenden Bereichen mit Wasser (indifferente Temperatur beachten!) durchnässt werden.

\section{Wärmestauender Wickel}

Der wärmestauende (kalte/warme), stoffwechselanregende Wickel findet bei chronisch-degenerativen 

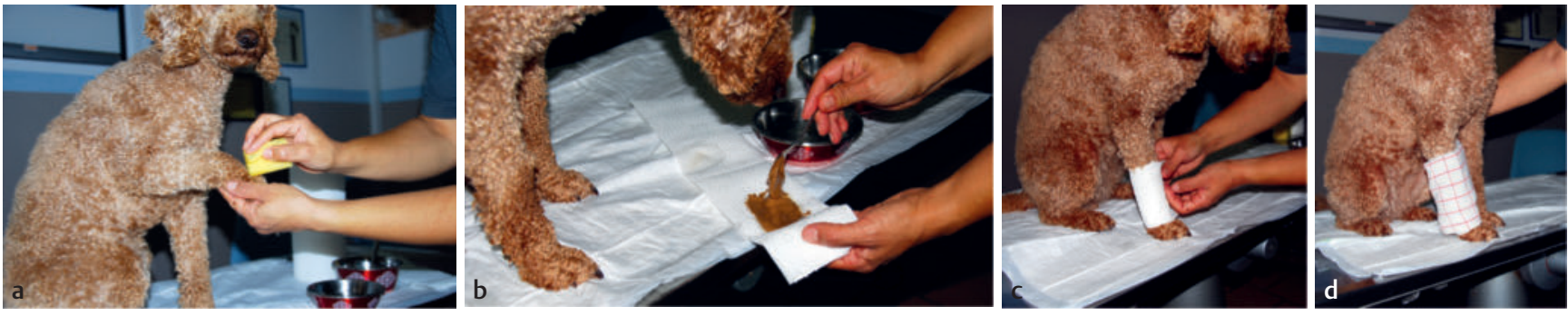

- Abb.4 Wärmeentziehender Gelenkwickel mit Heilerde. a Gelenk mit indifferent temperiertem Wasser nässen. b Das breiige Heilerdegemisch wird auf ein passgenau zugeschnittenes Nasstuch aufgetragen. Hierfür eignen sich z. B. Küchenrollen. c Das Nasstuch wird um die Gliedmaße gewickelt. d Abschließend mit einem Trockentuch (hierfür eignen sich Geschirrhandtücher oder Verbände) umwickeln. Quelle: @ Stefanie Süße, Reinhold Scharwey.

Gelenkerkrankungen (Arthrosen) Anwendung. Auch bei dieser Anwendung sollte der Bereich vorher aufgrund des isolierenden Felles mit Wasser indifferenter Temperatur durchnässt werden. Anschließend wird das Nasstuch wie beim Kaltwickel mit kaltem Wasser (bis zu $10^{\circ} \mathrm{C}$ unter der Temperatur des Behandlungsbereichs des Patienten) um das Behandlungsgebiet gewickelt, anschließend folgt das Trockentuch. Der Unterschied zum Kaltwickel besteht in der Länge der Anwendung desselben Wickels, d.h. der Wickel wird während der Anwendung nicht gewechselt, die Anwendung dauert 20-30 Minuten oder länger. Wichtig ist der gute seitliche Abschluss des Wickels, um eine Kaminwirkung zu vermeiden, bei der Zugluft dem Wärmestau entgegenwirkt. Chronische Gelenkerkrankungen können nach Anleitung und regelmäßiger Kontrolle auch vom Patientenbesitzer durchgeführt werden.

\section{Weitere Wickel}

Bei funktionellen Störungen der Leber oder Nieren können Leibwickel ( $\triangleright$ Abb.5) zum Einsatz kommen. Bei funktionellen Störungen der unteren luftführenden Atemwege wird der Brustwickel ( $\triangleright$ Abb.6) angewendet. Es sind auch Wickel mit Kartoffeln, Kohl, Leinsamen usw. möglich. Durch diese Zusätze ist die Temperaturübertragung langsamer als bei Wasser.

\section{CAVE}

Beinwell (Symphytum) und Senfmehl sind aufgrund möglicher starker Reizwirkungen beim Kleintier nicht zu empfehlen.

Die Wickel können grundsätzlich kalt oder warm angewendet werden. Warme Wickel haben keine vegetative Umstimmung zur Folge wie kalte Wickel es haben. Beim warmen Wickel wird die Wirkung von außen erreicht, bei kalten Wickeln muss der Körper die Wärme selbst erzeugen.

\section{Heiße Rolle}

Sie gestattet eine in weiten Grenzen abstufbare Dosierung von milden bis heißen Wärmegraden und somit eine weitgehende Anpassung an die individuelle Verträglichkeit und unterschiedliche Indikationsgebiete:

- muskuläre Syndrome

- chronische Entzündungen

- degenerative Gelenkveränderungen

- Wirbelsäulensyndrome

Zur Vorbereitung einer heißen Rolle wird ein Frottiertuch (ca. $50 \times 90 \mathrm{~cm}$ ) benötigt. Zunächst wird dieses der Länge nach gefaltet und anschließend zu einer festen Rolle aufgerollt. Diese Rolle wird leicht konisch gerollt, um an der offenen Seite einen Trichter zu erhalten. Der Trichter dient der Aufnahme des langsam eingegossenen heißen Wassers. Es wird so viel Wasser eingefüllt, bis die Rolle in der oberen Hälfte bis außen nass ist. Zur lokalen thermischen Anwendung werden die Körperabschnitte zunächst tupfend und später rollend behandelt. Dabei werden immer nur lokale Anwendungen durchgeführt. Man arbeitet mit der Rolle bis die behandelte Region gut erwärmt ist. Durch stetiges Abrollen der Frottierlagen kann die Behandlungstemperatur über längere Zeit konstant gehalten werden.

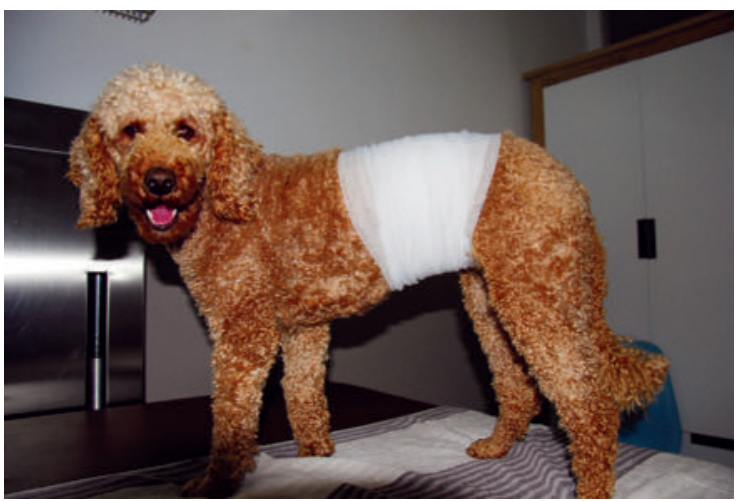

- Abb.5 Der Leibwickel wird nach dem Benässen des Felles mit indifferent temperiertem Wasser angelegt. Dazu wird das Nasstuch ohne komprimierende Wirkung locker, aber anliegend um das Abdomen gewickelt. AnschlieBend wird das Trockentuch in derselben Weise gewickelt. Quelle: @ Stefanie Süße, Reinhold Scharwey 


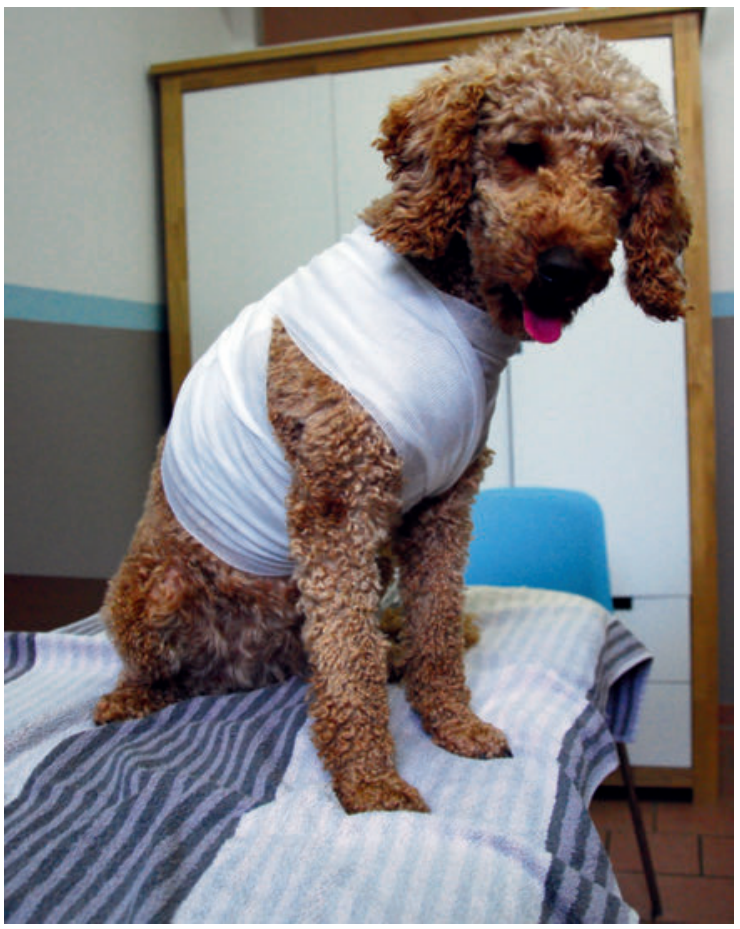

- Abb. 6 Das Wickeln des Brustwickels erfolgt wie beim Leibwickel (Fell durchnässen, Nasstuch, Trockentuch). Das Behandlungsgebiet erstreckt sich um den Thorax mit der Begrenzung nach kaudal auf Höhe der letzten Rippe. Zu beachten ist eine kreuzende Wickelung durch die Vorderbeine bis zum unteren Halsabschnitt, da sonst der kraniale Abschnitt des Thorax ausgespart bliebe. Quelle: ( Stefanie Süße, Reinhold Scharwey

\section{Eisanwendungen}

Die optimale Temperatur für einen Eislolli sollte $-4^{\circ} \mathrm{C}$ (Temperatur des Gefrierfachs eines Kühlschranks) nicht unterschreiten. Der Einsatz von Eisanwendungen beschränkt sich auf frühzeitige posttraumatische Anwendungen innerhalb der vaskulären Entzündungsphase, um die Entzündung zu hemmen und ggf. die Schmerzen zu reduzieren. Das Eis kann zudem tupfend angewendet zur Stimulation bei neurologischen Erkrankungen mit Paresen und verminderter Sensibilität genutzt werden.

\section{CAVE}

Besondere Vorsicht ist bei Patienten mit Hyperästhesien geboten.

\section{Dampfanwendungen (Inhalationen)}

Grundsätzlich unterscheiden sich die Wirkungen der Dampfanwendungen oder Inhalationen in der eingebrachten Tröpfchengröße. Bei einfachen Verdampfungen werden die oberen Atemwege inkl. des Hauptbronchus angesprochen. Um tiefere Atemwege zu erreichen, müssen die Lösungen, z. B. Salzwasser, zur Inhalation über im Handel erhältliche Geräte mittels Ultraschall vernebelt werden.

\section{Kontraindikationen}

Die Kontraindikationen der Hydrotherapie sind je nach Ausmaß, Stärke der Erkrankung und Situation des Tieres sowie der ausgewählten Anwendungsform zu beachten. So beeinflusst eine lokal angewendete Kompresse den Kreislauf weniger stark als beispielsweise ein Leibwickel.

Absolute Kontraindikationen der Hydrotherapie:

- dekompensierte Herz-Kreislauf-Erkrankungen

- lokale offene Wunden im Behandlungsgebiet

- Fieber (Ausnahme temperatursenkende Wadenwickel)

- systemische Infektionen

- maligne Tumore

Relative Kontraindikationen der Hydrotherapie:

- Herzerkrankungen

- lokale Infektionen

- Epilepsie

- trächtige Hündinnen

Leichtere Formen der Herzerkrankungen sowie gut eingestellte Herzpatienten oder Epileptiker stellen normalerweise keine Kontraindikation dar. Die Patienten sollten während der Anwendung aufmerksam beobachtet werden. Kommt es bei einem Epilepsiepatienten zeitlich im Zusammenhang zur Anwendung zu einem Anfall, so ist die Hydrotherapie anzupassen, zu überdenken und ggf. abzubrechen. Es empfiehlt sich, die Therapie in oben genannten Fällen mit milden Reizen (handwarmes Wasser) einzuleiten.

\section{Take home}

Die klassische Hydrotherapie bietet vielfältige Möglichkeiten von Teilbädern bis hin zu lokalen Wickeln, um einen positiven Einfluss auf einen Organismus auszuüben. Dabei können Effekte des hydrostatischen Druckes, der Auftriebskraft, des Reibungswiderstands, Temperaturen, Leitfähigkeiten der Medien usw. ausgenutzt werden. Eine Anregung des Stoffwechsels wird bei den Kneipp‘schen Güssen erzielt. Entzündungshemmung und Stoffwechselanregung spielen eine wichtige Rolle bei der Anwendung von Wickeln in der Therapie von
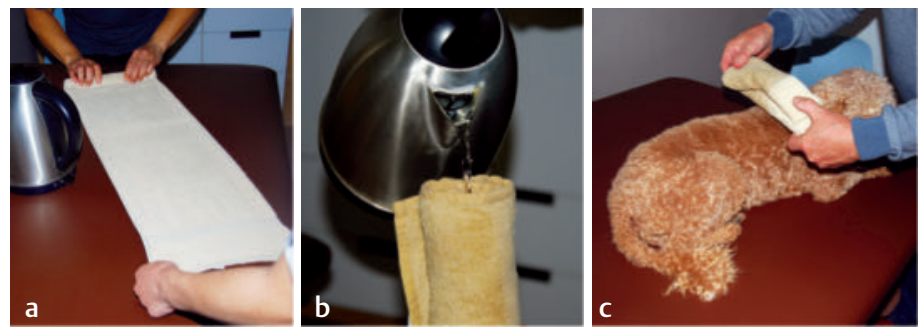

- Abb. 7 Heiße Rolle beim Hund. a Falten und aufwickeln des Handtuchs. b Heiße Rolle mit kochendem Wasser befüllen. c Abtupfen und abrollen des Rückens mit der heißen Rolle. Quelle: @ Stefanie Süße, Reinhold Scharwey 
Gelenkerkrankungen. Während der Hydrotherapie sollte der Patient sorgfältig beobachtet werden, um Kreislaufbelastungen, Unwohlsein, ggf. auch Schmerz rechtzeitig erkennen zu können.

\section{Autoren}

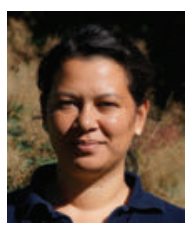

\section{Stefanie Süße}

Tierärztin und Dozentin für Physikalische Medizin und Physiotherapie beim Kleintier im Vierbeiner Reha-Zentrum in Bad Wildungen; Spezialgebiete: Physikalische Medizin und Physiotherapie beim Kleintier, Traditionelle Chinesische Medizin, Goldimplantation und Osteopathie beim Hund; ssuesse@vierbeiner-rehazentrum.de

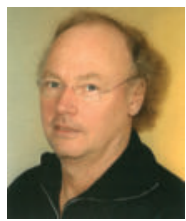

\section{Reinhold Scharwey}

examinierter Masseur, Bademeister und Humanphysiotherapeut; Tierphysiotherapeut (Bad Wildungen); Spezialgebiete: Manuelle Lymphdrainage, Elektrotherapie und Interferenzstrom-Regulationstherapie (IFR); seit 2004 Dozent für Physikalische Medizin und Physiotherapie beim Kleintier im Vierbeiner RehaZentrum in Bad Wildungen; scharwey@vierbeiner-rehazentrum.de
Literatur

[1] Alexander CS. Hydrotherapie und Laufbandtraining. Grundlagen und Trainingskonzepte für Hunde. Hannover: Schlütersche; 2019

[2] Baumgarten A. Die Kneipp‘sche Hydrotherpie. Wörishofen: Buchdruckerei und Verlags-Anstalt; 1909

[3] Bockstahler B, Millis D, Levine D. Physiotherapie auf den Punkt gebracht. Rehabilitation und Schmerzmanagement. Babenhausen: BE VetVerlag; 2004

[4] Gillert O. Hydrotherapie und Balneotherapie in Theorie und Praxis. 7. Aufl. München: Richard Pflaum; 1978

[5] Holstege L. Die Hydrotherapie beim Pferd. Geschichte und heutige Bedeutung [Dissertation]. Hannover: Stiftung Tierärztliche Hochschule Hannover; 2019

[6] Vierbeiner Reha-Zentrum GmbH. Physikalische und Rehabilitative Medizin beim Kleintier [Kursskript]. Bad Wildungen: Vierbeiner Reha-Zentrum GmbH; 2019

Bibliografie

DOI https://doi.org/10.1055/a-1017-2969

Hands on 2019; 1: 14-20

(c) Georg Thieme Verlag KG Stuttgart · New York ISSN 2628-6033 